BNL-112201-2016-JA

\title{
Mini Compton Camera Based on an Array of Virtual Frisch-Grid CdZnTe Detectors
}

Wonho Lee, Aleksey Bolotnikov, Taewoong Lee, Giuseppe Camarda, Yonggang Cui, Rubi Gul, Anwar Hossain, Roy Utpal, Ge Yang, and Ralph James

Submitted to IEEE TRANSACTIONS ON NUCLEAR SCIENCE

February 2016

Nonproliferation and National Security Department

Brookhaven National Laboratory

\author{
U.S. Department of Energy \\ USDOE National Nuclear \\ Security Administration (NNSA), \\ Office of Nonproliferation and \\ Verification Research and Development (NA-22)
}

Notice: This manuscript has been authored by employees of Brookhaven Science Associates, LLC under Contract No. DE- SC0012704 with the U.S. Department of Energy. The publisher by accepting the manuscript for publication acknowledges that the United States Government retains a non-exclusive, paid-up, irrevocable, world-wide license to publish or reproduce the published form of this manuscript, or allow others to do so, for United States Government purposes. 


\section{DISCLAIMER}

This report was prepared as an account of work sponsored by an agency of the United States Government. Neither the United States Government nor any agency thereof, nor any of their employees, nor any of their contractors, subcontractors, or their employees, makes any warranty, express or implied, or assumes any legal liability or responsibility for the accuracy, completeness, or any third party's use or the results of such use of any information, apparatus, product, or process disclosed, or represents that its use would not infringe privately owned rights. Reference herein to any specific commercial product, process, or service by trade name, trademark, manufacturer, or otherwise, does not necessarily constitute or imply its endorsement, recommendation, or favoring by the United States Government or any agency thereof or its contractors or subcontractors. The views and opinions of authors expressed herein do not necessarily state or reflect those of the United States Government or any agency thereof. 


\title{
Mini Compton Camera Based on an Array of Virtual Frisch-Grid CdZnTe Detectors
}

\author{
Wonho Lee, Aleksey Bolotnikov, Taewoong Lee, Giuseppe Camarda, Yonggang Cui, \\ Rubi Gul, Anwar Hossain, Roy Utpal, Ge Yang, and Ralph James
}

\begin{abstract}
We constructed a mini Compton camera based on an array of CdZnTe detectors and assessed its spectral and imaging properties. The entire array consisted of $6 \times 6$ Frisch-grid CdZnTe detectors, each with a size of $6 \times 6 \times 15 \mathrm{~mm}^{3}$. Since it is easier and more practical to grow small CdZnTe crystals rather than large monolithic ones, constructing a mosaic array of parallelepiped crystals can be an effective way to build a more efficient, large-volume detector. With the fully operational CdZnTe array, we measured the energy spectra for ${ }^{133} \mathrm{Ba}-,{ }^{137} \mathrm{Cs}-$, ${ }^{60} \mathrm{Co}$-radiation sources; we also located these sources using a Compton imaging approach. Although the Compton camera was small enough to hand-carry, its intrinsic efficiency was several orders higher than those generated in previous researches using spatially separated arrays, because our camera measured the interactions inside the CZT detector array, wherein the detector elements were positioned very close to each other. The performance of our camera was compared with that based on a pixelated detector.
\end{abstract}

Index Terms-CdZnTe, Compton camera, electronic collimation, Frisch-grid.

\section{INTRODUCTION}

$\mathrm{CdZnTe}$ detectors have been used widely for measuring radiation energy because of their relatively high energy-resolution, high atomic number, and operability at room temperature [1]. However, due to the low mobility of holes in the material and its non-uniformity, the usability of these crystals for planar gamma-detectors has been limited; hence, special designs were developed to ensure their suitability for field-portable gamma-ray spectrometers, such as the co-planar grid [2], the small pixel structure [3], [4], and the virtual Frisch-grid device [5]-[9]. In current technology, the achievable size of a single crystal with high energy resolution is about $6 \mathrm{~cm}^{3}$

Manuscript received October 20, 2014; revised June 28, 2015; accepted December 26, 2015. Date of current version February 16, 2016. This work was supported by the U.S. Department of Energy , Office of Defense Nuclear Nonproliferation Research \& Development, DNN R\&D, U.S. Defense Threat Reduction Agency (DTRA), and BNL's Technology Maturation Award. The manuscript has been authored by Brookhaven Science Associates, LLC under Contract No. DE-AC02-98CH1-886 with the U.S. Department of Energy. This work was supported by National Research Foundation of Korea (NRF) grant (2015M2A2A4021766), funded by the Korean government (MEST).

$\mathrm{W}$. Lee and T. Lee are with the Department of Bio-Convergence Engineering, Korea University, Seoul 136-701, Korea (e-mail: wonhol@korea.ac.kr).

A. Bolotnikov, G. Camarda, Y. Cui, R. Gul, A. Hossain, R. Utpal, G. Yang, and R. James are with the Department of Nonproliferation and National Security, Brookhaven National Laboratory, Upton, NY 11973, USA.

Color versions of one or more of the figures in this paper are available online at http://ieeexplore.ieee.org.

Digital Object Identifier 10.1109/TNS.2015.2514120
[10]. Hence, to construct a large-volume $\mathrm{CdZnTe}$ detector suitable for compact, inexpensive instruments, we developed an assembly of detector modules using Frisch-grid devices [11]-[14], and a recently developed large array [15] was used for this research. The Frisch-grid device has a unique aspect in that it is simpler than multi-pixel approaches. Instead of the complex grids in multi-pixel devices requiring many channels of read-out, the Frisch-grid uses simple, robust electrodes and a small number of read-out channels per detector element for low-power consumption, while attaining comparable performance to multi-pixel devices. The shape of these devices is ideal to build blocks for assembling arrays, which can provide a larger effective area, good energy resolution, and good spatial resolution at comparatively low production cost compared to monolithic detectors. Therefore, the size of our assembly is not limited by the size of a single crystal, and it can be increased by merely adding elements or modules. By assembling $\mathrm{CdZnTe}$ modules, we developed a mini-gamma camera without requiring any additional instruments. The $\mathrm{CdZnTe}$ modules measured and yielded information on the energy and position of sequential interactions inside the camera; we calculated the original position of the radiation sources by applying Compton imaging technology to the measured information.

The feasibility of Compton imaging technology was proven several decades ago [16], [17], and it has been used in various instruments to reconstruct radiation sources. In the early stages of this research, most detector materials used for Compton imaging were gases or scintillators that could be present in a large volume and coupled with position-sensing devices [16]. With the improvements in semiconductor manufacturing and steady advances in crystal growth, a Compton camera fabricated from semiconductors became available, as well as scintillators [18]-[22]. Compared to gases and scintillators, semiconductors show high energy-resolution so assuring fine energy selectivity in the reconstructed Compton images. Semiconductors can also attain finer resolution in positioning than even their own pixel dimensions by using the timing information, or the signal ratio of different electrodes (i.e., charge-sharing characteristics), which improves the angular resolution of the reconstructed Compton image. There have been several research investigations on Compton cameras using one or more $\mathrm{CdZnTe}$ detectors, mostly with detectors relying on single-crystal geometry [18]-[20].

In this study, we discuss the performance of our Compton camera, consisting of a mosaic array of parallelepiped $\mathrm{CdZnTe}$ crystals; they have high potential to be built as a large-volume 

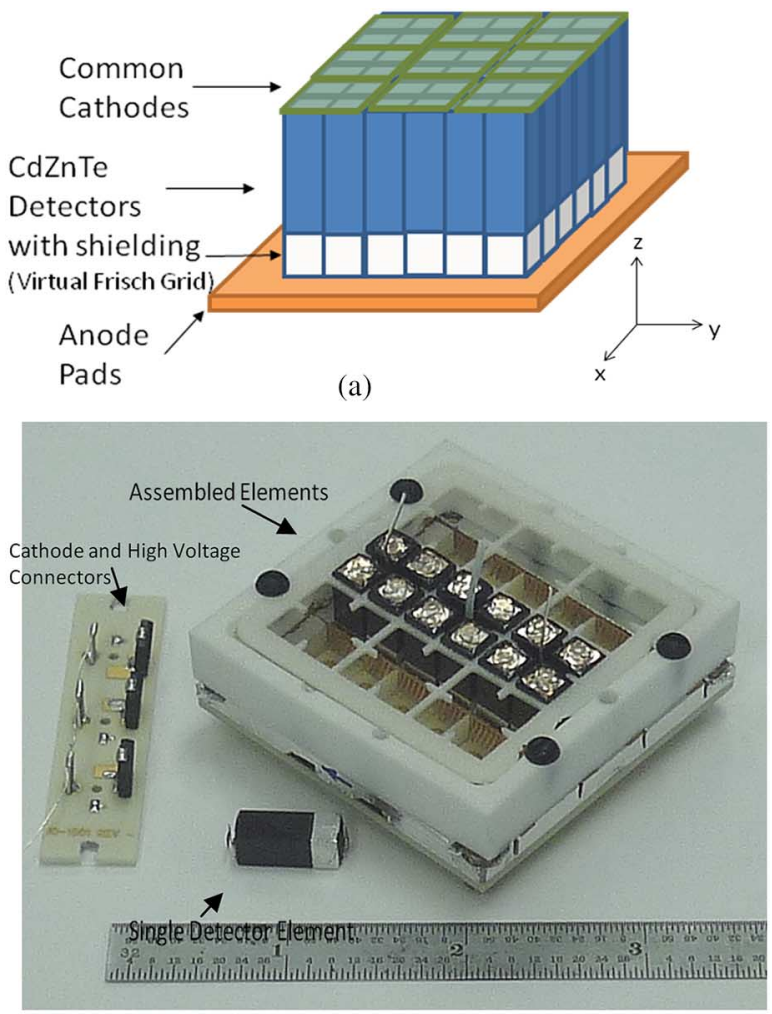

(b)

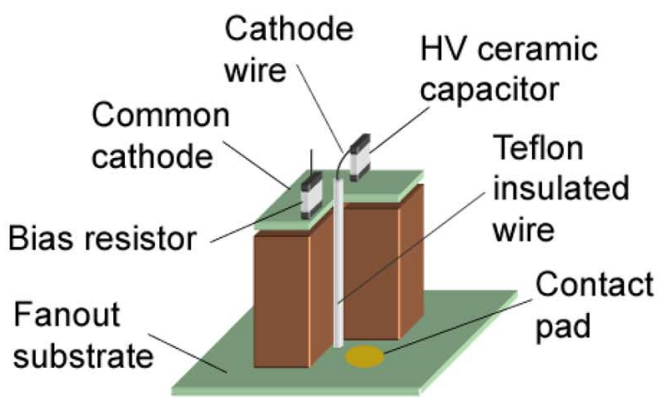

(c)

Fig. 1. (a) Schematic diagram of a $6 \times 6$ mosaic array of parallelepiped CdZnTe crystals with electrodes. (b) Photograph of the detector array for a mini Compton camera, illustrating a single detector element, and the partially assembled elements in it. (c) Schematic diagram of 3 elements in a $2 \times 2$ sub-array.

detector array, resulting in an increase in the efficiency of the detection, and the availability to apply mechanical collimation requiring a planar sensing area $\left(>10 \mathrm{~cm}^{2}\right)$ with high energy and position- resolution. Our Compton camera is applicable as a portable imager to monitor nuclear materials in nuclear power plants and nuclear waste in reservoirs.

\section{Materials AND Methods}

Fig. 1(a) shows a schematic diagram of a mosaic detector array for a mini Compton camera. Fig. 1(b) is a photograph of an individual element and an entire array partially filled by different Frisch-grid detectors. Fig. 1(c) is the corresponding schematic diagram of a $2 \times 2$ sub-array in a mini Compton camera [15]. The assembly consisted of $6 \times 6 \mathrm{CdZnTe}$ detectors, each of which has a volume of $6 \times 6 \times 15 \mathrm{~mm}^{3}$; the gap between them was $1.5 \mathrm{~mm}$. A bias resistor and a $\mathrm{HV}$ capacitor are a decoupling circuitry to selectively pass the pulse signal from a CdZnTe crystal to electronics and protect the electronics from high voltage. Based on the cathode-to-anode ratio and drift time, we corrected the information on the energy of the radiation interaction, so that we could calculate the depth-of-interaction position between the cathode and anode [14]. Therefore, the $\mathrm{z}$ positions of radiation interactions could be determined at sub-crystal resolution, while the position resolutions in $\mathrm{x}$ and $y$ directions were limited by the finite size of the crystal. The position resolution in the $\mathrm{z}$ direction (depth direction) was less than $0.5 \mathrm{~mm}$ based on the drift-time measurements. The coincidence window of the electronics can be set as low as $20 \mathrm{~ns}$. We note that this was a prototype system, and its electronics could handle more than a tenfold increase in the number of detectors for further studies.

Since the position and energy information for each radiation interaction in the detectors are known, the location of the radiation source can be identified via known Compton imaging technology. If the position and energy information of the source, $1 \mathrm{st}$ interaction and 2nd interaction, respectively, are $\left(r_{0}, E_{0}\right),\left(r_{1}\right.$, $\left.E_{1}\right)$, and $\left(r_{2}, E_{2}\right)$; the cosine values can be calculated based on this information as follows:

$$
\begin{aligned}
& \cos \theta_{r}=\frac{\bar{r}_{1} \cdot \bar{r}_{2}}{\left|\bar{r}_{1}\right|\left|\bar{r}_{2}\right|} \\
& \overline{r_{1}}: \text { vector from } r_{0} \text { to } r_{1} \\
& \overline{r_{2}}: \text { vector from } r_{1} \text { to } r_{2} \\
& \cos \theta_{E}=1-m_{0} c^{2}\left(\frac{1}{E_{2}}-\frac{1}{E_{1}}\right) \\
& m_{0} c^{2}: \text { the rest mass of the electron. }
\end{aligned}
$$

Equalizing the two cosine values, we then can calculate the source's position, $\mathrm{r}_{0}$, based on the positions $\left(r_{1}\right.$ and $\left.r_{2}\right)$ and the energy information $\left(E_{1}\right.$ and $\left.E_{2}\right)$ for the two photon interactions measured by the detector elements in the array. Based on these above equations, we can draw a cone on the source space for each interaction event. Fig. 2 (upper) illustrates how to draw a cone containing a source position. With the position and energy information of two interactions, a cone can be projected in a spherical coordinate system. We assume that the source is located along the exterior surface of the cone. Three and more sequential interactions are disregarded for image reconstruction. If we measure multiple events, the overlaps of the cones will indicate the source position (cf. Fig. 2 (lower)). Since the position resolution of our detector is relatively high in $\mathrm{z}$ direction but low in the $\mathrm{x}$ and $\mathrm{y}$ directions, if a radiation is vertically incident on the top of the detector, the positional measurement of the scattered radiation with large scattering angle can be more precise than that with small scattering angle.

Since the overlaps of the cones are a simple back-projection, including inherent angular broadening, additional image reconstruction is required to precisely locate the source's position. We improved the angular resolution of the reconstructed images using the maximum likelihood expectation and maximization (MLEM) method, as shown in (3). This statistical reconstruction algorithm, including the Poisson distribution, is widely used in 

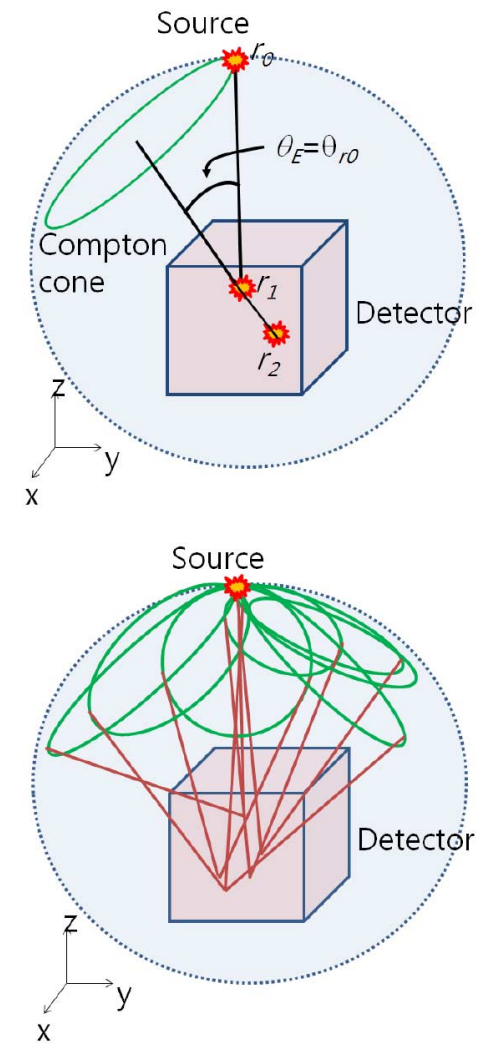

Fig. 2. Schematic diagram of Compton imaging for a single (upper) and multiple (lower) events.

Compton imaging due to its effectiveness for obtaining the reconstructed image with limited number of radiation-event measurements [23]-[25].

$$
x_{j}^{n+1}=\frac{x_{j}^{n}}{\sum_{i} c_{i j}} \sum_{i} \frac{c_{i j} Y_{i}}{\sum_{k} c_{i k} x_{k}^{n}}
$$

where $x_{j}^{n+1}$ and $x_{j}^{n}$ are $(\mathrm{n}+1)$ th and nth estimates of pixel values at the source plane, where the initial values, $x_{j}^{0}$, all are positive. $c_{i j}$ is the probability of ith detection event for an emitted photon at the jth source pixel. $c_{i j}$ includes the angular probabilistic distribution of Compton scattering calculated by the Klein-Nishina formula, and the radiation attenuations in the detector divided by the square of the distances from the source plane to the 1 st interaction point, and from that point to the 2 nd interaction point. $c_{i j}$ is analytically calculated for each event, which includes the direction and distance of the photon transport and interaction in the detector. The precision of the outcome using the Klein-Nishina formula and the amount of attenuation is limited by the energy resolution of the detector.

The positional resolution of the interaction points in the $x$ and y directions was limited by the finite size of the crystal, and that in the $\mathrm{z}$ direction was limited by the finite resolution of drift time. $Y_{i}$ is the measured data for the detection event, i. In Compton imaging reconstruction, the total number of possible combinations of the position- and energy- measurements was significantly larger than that of the detected photons needed to be considered, which requires a long calculation time and excessive data storage for a conventional computer. Therefore, instead we applied a list-mode MLEM. In the list mode, each measurement including the position and energy information is unique rather than adding a count to a position and energy bin. Each unique measurement is like a single point in the continuous measurement space, and hence, the measured count for every unique event is one (cf. $Y_{i}=1$ ) [25]. Since the total number of detected events is much smaller than that of all possible combination of position- and energy- measurements, the matrix size for image processing dramatically decreases, and the calculation time is reduced as much.

The sequence of the interactions was determined by the energy of each one if the cosine angle calculated by equalizing Equations (1) and (2) was acceptable. According to Y. F. Du et al. [18] and W. Lee et al. [26], if the energy of the incident gamma ray was less than $400 \mathrm{keV}$, it is more probable that the first interaction deposits less energy than does the second interaction in two-event sequences, and vice versa. Therefore, for a 356-keV gamma-ray, we chose the less energetic events as the first interaction, while the higher energy events were the first interactions for higher energy gamma-rays (i.e., 662-, and $1275-\mathrm{keV})$. The incorrect determination for the sequence of radiation interaction is more probable when the incident radiation energy is close to $400 \mathrm{keV}$, which causes artifacts in the reconstructed image especially in the opposite direction of the original source position.

Experimental setups were used to evaluate the performance of the mini Compton camera. First, point sources of $169.5-\mathrm{kBq}$ ${ }^{133} \mathrm{Ba}(356 \mathrm{keV}), 281.6-\mathrm{kBq}{ }^{137} \mathrm{Cs}(662 \mathrm{keV})$ and $7.8-\mathrm{kBq}$ ${ }^{60} \mathrm{Co}(1173,1332 \mathrm{keV})$ were located $14.5 \mathrm{~cm}$ from the top of the detector array. The measurement times for ${ }^{133} \mathrm{Ba}$ and ${ }^{137} \mathrm{Cs}$ were 2 hours, and that for ${ }^{60} \mathrm{Co}$ was 10 hours. In our second experiment, point sources of $169.5-\mathrm{kBq}{ }^{133} \mathrm{Ba}, 281.6-\mathrm{kBq}$ ${ }^{137} \mathrm{Cs}$, and $7.8-\mathrm{kBq}{ }^{60} \mathrm{Co}$ were positioned at the vertexes of an equilateral triangle, and the gamma-rays from all three sources were detected simultaneously. The plane with the multiple sources was placed $17 \mathrm{~cm}$ from the detector, and the distance between sources was $14.5 \mathrm{~cm}$. The measurement time was 2 hours. The background dose rate was $29.8 \mu \mathrm{R} / \mathrm{hr}$.

\section{RESUlts}

The energy resolutions of the spectra for single events were measured by each detector element. The resolutions were calculated at the full-width-half-maximum (FWHM) divided by the peak channel for $662-\mathrm{keV}$ gamma rays. The energy resolution for all detector elements was $1.08 \%$. The mean and standard deviation of the energy resolution for individual detectors was $1.07 \%$ and $\pm 0.30 \%$.

The energy resolutions of the coincident events for ${ }^{133} \mathrm{Ba}$ $(356 \mathrm{keV}),{ }^{137} \mathrm{Cs}(662 \mathrm{keV})$, and ${ }^{60} \mathrm{Co}(1173,1332 \mathrm{keV})$, respectively, were 1.96-, 1.36-, 1.19-, and 1.13-\%. The energy resolution of the coincident events for a ${ }^{137} \mathrm{Cs}$ source was broader than that of the single events, because the energy resolution is inversely proportional to the deposited energy, and the individually deposited energies of a coincident event are lower than that of a single event. In addition, the electronic noise increases when each of the electronic signals induced by a sequential interaction associated with a Compton scattering followed by a 


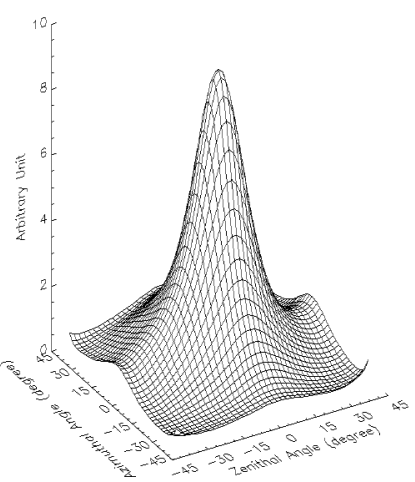

(a)

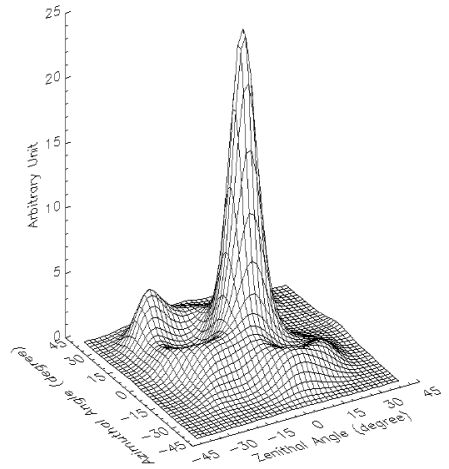

(b)
Fig. 3. Reconstructed images of a $662 \mathrm{keV}$ point source with MLEM method (a) 10th iteration and (b) 100th iteration.

photoelectric effect is summed. The peak-to-total ratios of the spectra for coincident events were 2.7 times higher than that for single events, because most of the Compton continuum consists of single Compton scattering events, followed by the escape of the scattered gamma rays, whilst the photo-peak can be composed of coincident events, such as Compton scatterings followed by photoelectric events [27].

Based on the measured energy spectrum of the coincidence events, the photo-peaks for incident radiation energies were identified. By energy windowing around the peaks, the events composed of a Compton scattering followed by a photoelectric event were selectively chosen for image reconstruction.

The angular resolution measurement (ARM) is the difference between the angles calculated based on the position and energy information (cf. (1) and (2)) when the location of the source was known as the center of the field-of-view (FOV). The FWHM of the ARM for ${ }^{133} \mathrm{Ba}(356 \mathrm{keV}),{ }^{137} \mathrm{Cs}(662 \mathrm{keV})$, and ${ }^{60} \mathrm{Co}$ $(1173,1332 \mathrm{keV})$, respectively were $30.9^{\circ}, 32.9^{\circ}, 22.5^{\circ}$ and $21.2^{\circ}$. The full width tenth maximum (FWTM) of the ARM for ${ }^{133} \mathrm{Ba}(356 \mathrm{keV}),{ }^{137} \mathrm{Cs}(662 \mathrm{keV})$, and ${ }^{60} \mathrm{Co}(1173,1332 \mathrm{keV})$, respectively, were $92.1^{\circ}, 84.8^{\circ}, 71.0^{\circ}$, and $55.6^{\circ}$. Since angular uncertainty is related to energy uncertainty, and energy resolution is inversely proportional to the square root of the incident energy, the FWHM and FWTM of the ARM generally decreased proportionally with the increase of the incident radiation energy. The width of each back-projection cone for Compton imaging (cf. Fig. 2) was set based on that of the ARM.

Fig. 3 showed the image of a point ${ }^{137} \mathrm{Cs}$ source reconstructed using the MLEM method. Only when the combined energy of two sequential events was in the range of the photo-peak region for each source, the events were selected for Compton reconstruction. Fig. 4 showed the change of FWHMs with iteration. As discussed in previous studies [19], [28], the reconstructed image was sharper and had more noise with higher iteration. We identified artifacts, as predicted to be caused by the incorrect determination of the interaction sequence, in the reconstructed image especially at the opposite direction of the original point source.

Table I summarizes the FWHMs, intrinsic efficiency, and absolute efficiency for each radiation source at $14.5 \mathrm{~cm}$ from the detector. The intrinsic imaging efficiency was the ratio of the

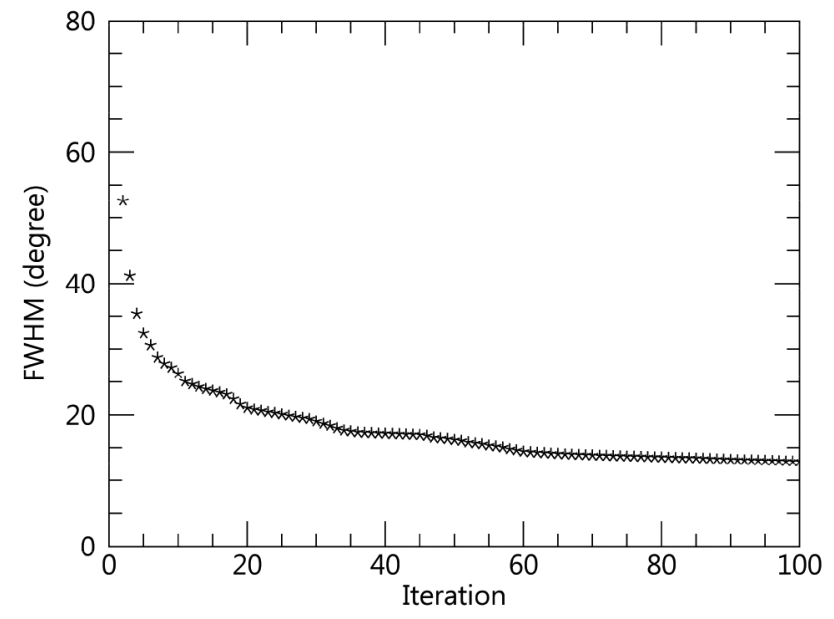

Fig. 4. FWHM vs. number of iterations.

TABLE I

MEASUREMENT RESUltS FOR VARIOUS GAMMA-RAy ENERGIES

\begin{tabular}{|c|c|c|c|c|c|c|}
\hline \multirow{2}{*}{$\begin{array}{c}\text { Energy } \\
(\mathrm{keV})\end{array}$} & \multirow{2}{*}{$\begin{array}{c}\text { Energy } \\
\text { Resolution } \\
(\%)\end{array}$} & \multicolumn{3}{|c|}{$\begin{array}{l}\text { FWHM } \\
\text { (degree) }\end{array}$} & \multicolumn{2}{|c|}{ Efficiency } \\
\hline & & ARM & $10^{\text {th }}$ & $100^{\text {th }}$ & Intrinsic & $\begin{array}{l}\text { Absolute } \\
(14.5 \mathrm{~cm})\end{array}$ \\
\hline 356 & $\begin{array}{c}1.96 \\
\pm 0.08\end{array}$ & $\begin{array}{r}30.9 \\
\pm 0.8\end{array}$ & $\begin{array}{r}30.4 \\
\pm 1.0\end{array}$ & $\begin{array}{r}18.2 \\
\pm 1.9\end{array}$ & $\begin{array}{c}(1.81 \\
\pm 0.05) \\
\times 10^{-2}\end{array}$ & $\begin{array}{c}(8.73 \\
\pm 0.24) \\
\times 10^{-5}\end{array}$ \\
\hline 662 & $\begin{array}{c}1.36 \\
\pm 0.04\end{array}$ & $\begin{array}{r}32.9 \\
\pm 0.8\end{array}$ & $\begin{array}{l}26.0 \\
\pm 1.6\end{array}$ & $\begin{array}{r}13.9 \\
\pm 1.8\end{array}$ & $\begin{array}{c}(0.94 \\
\pm 0.02) \\
\times 10^{-2} \\
\end{array}$ & $\begin{array}{c}(4.53 \\
\pm 0.11) \\
\times 10^{-5} \\
\end{array}$ \\
\hline 1173 & $\begin{array}{c}1.19 \\
\pm 0.08\end{array}$ & $\begin{array}{r}22.5 \\
\pm 1.0\end{array}$ & $\begin{array}{c}20.3 \\
\pm 1.3\end{array}$ & $\begin{array}{r}12.6 \\
\pm 1.0\end{array}$ & $\begin{array}{c}(0.50 \\
\pm 0.02) \\
\times 10^{-2}\end{array}$ & $\begin{array}{c}(2.42 \\
\pm 0.11) \\
\times 10^{-5}\end{array}$ \\
\hline 1332 & $\begin{array}{c}1.13 \\
\pm 0.08\end{array}$ & $\begin{array}{r}21.2 \\
\pm 1.1\end{array}$ & $\begin{array}{c}19.1 \\
\pm 0.9\end{array}$ & $\begin{array}{r}12.6 \\
\pm 1.0\end{array}$ & $\begin{array}{c}(0.44 \\
\pm 0.02) \\
\times 10^{-2}\end{array}$ & $\begin{array}{c}(2.12 \\
\pm 0.11) \\
\times 10^{-5}\end{array}$ \\
\hline
\end{tabular}

TABLE II

COMPARISON OF EXPERIMENT AND SIMULATION RESULTS

\begin{tabular}{|c|c|c|c|c|}
\hline \multirow{2}{*}{$\begin{array}{c}\text { Energy } \\
(\mathrm{keV})\end{array}$} & \multicolumn{2}{|c|}{$\begin{array}{c}\text { FWHM at } 10^{\text {th }} \text { iteration } \\
\text { (degree) }\end{array}$} & \multicolumn{2}{|c|}{ Intrinsic Efficiency } \\
\hline & Experiment & Simulation & Experiment & Simulation \\
\hline 356 & $30.4 \pm 1.0$ & $27.0 \pm 0.06$ & $\begin{array}{c}(1.81 \pm 0.05) \\
\times 10^{-2}\end{array}$ & $\begin{array}{c}(1.86 \pm 0.006) \\
\times 10^{-2}\end{array}$ \\
\hline 662 & $26.0 \pm 1.6$ & $23.3 \pm 0.02$ & $\begin{array}{c}(0.94 \pm 0.02) \\
\times 10^{-2}\end{array}$ & $\begin{array}{c}(1.29 \pm 0.007) \\
\times 10^{-2}\end{array}$ \\
\hline 1173 & $20.3 \pm 1.3$ & $19.2 \pm 0.03$ & $\begin{array}{c}(0.50 \pm 0.02) \\
\times 10^{-2}\end{array}$ & $\begin{array}{c}(0.78 \pm 0.009) \\
\times 10^{-2}\end{array}$ \\
\hline 1332 & $19.1 \pm 0.9$ & $18.1 \pm 0.10$ & $\begin{array}{c}(0.44 \pm 0.02) \\
\times 10^{-2}\end{array}$ & $\begin{array}{c}(0.66 \pm 0.010) \\
\times 10^{-2}\end{array}$ \\
\hline
\end{tabular}

number of effective events (coincident events whose summed energy was in the energy window around the photo-peaks) used for image reconstruction to the total number of gamma rays incident on the surface of the detector; the absolute efficiency was the ratio of the number of the same effective events to the total number of gamma rays emitted from the source.

Table II shows the comparison of the experimental and simulation results. The simulation tool was Monte Carlo N Particle eXtended (MCNPX) code. The simulation geometry included an Al box containing the detector array, plastic layers between CZT crystals, and PCB boards at the backside of the detector 


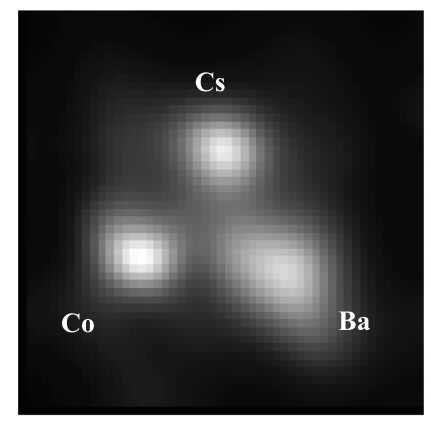

(a)

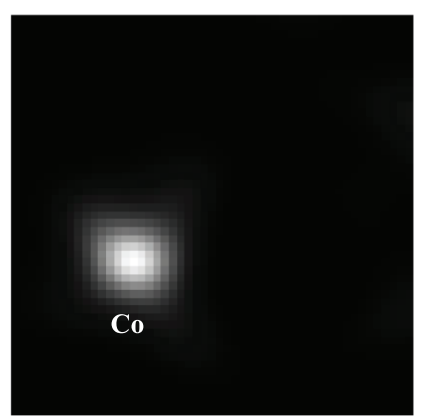

(c)

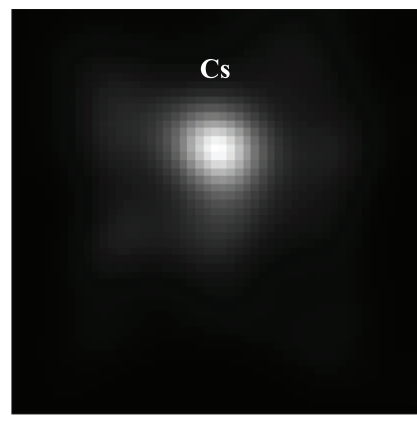

(b)

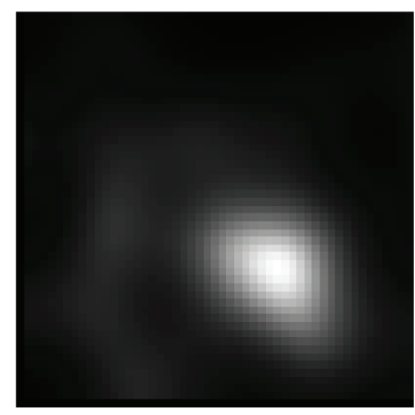

(d)
Fig. 5. Reconstructed images of multiple sources with MLEM method (50th iteration). (a) All three sources (b) Cs (662 keV), (c) Co (1173 and $1332 \mathrm{keV})$, and (d) $\mathrm{Ba}(356 \mathrm{keV})$.

array. The angular resolutions of the reconstructed images in experiment were well matched with those in simulation, and the trend of the intrinsic efficiencies measured by experiments followed with that calculated using simulations. The difference of the experimental and simulated results could be caused by the inclusion of factors such as electronic noise, energy threshold, energy window, dead time and dead layer in crystal. According to the simulation, our Compton imager could operate for the incident energy ranged from $200 \mathrm{keV}$ up to at least $3 \mathrm{MeV}$. If the energy is lower than $200 \mathrm{keV}$, the photoelectric effect is more dominant than Compton scattering, and the energy uncertainty is significantly higher. As a result, the detection efficiency of effective events is low, and the angular uncertainty in the reconstructed image is excessively high. If the energy is higher than several $\mathrm{MeV}$, the probability of a Compton scattering followed by a photoelectric effect is significantly lower, and hence, the detection efficiency of effective events becomes minimal.

Fig. 5 shows the reconstructed images of multiple sources after the 50th iteration. Every source, which was measured simultaneously, was clearly identified in each of its own positions. In Fig. 5(a), multiple sources were demonstrated by combining the individually reconstructed images (cf. Figs. 5(b)-5(d)) of three sources measured simultaneously. Before combination, each image was normalized by the peak count of the image. The radius of each reconstructed source was inversely proportional to the energy of the gamma rays emitted from the source, which was consistent with the results in Table I.

Table III shows the comparison of performances between our camera and a pixelated CZT detector [19]. The pixelated CZT detector composed of a single $15 \mathrm{~mm} \times 15 \mathrm{~mm} \times 10 \mathrm{~mm}$ CZT detector with $11 \times 11$ pixels. The size of the pixels was smaller
TABLE III

Comparison of the Performance for Two Detection Systems

\begin{tabular}{|c|c|c|}
\hline \multirow[b]{2}{*}{$\begin{array}{l}\text { Performance to detect } \\
\qquad 662 \mathrm{keV}\end{array}$} & \multicolumn{2}{|c|}{ Compton cameras $\left(662 \mathrm{keV}, 10^{\text {th }}\right.$ iteration) } \\
\hline & $\begin{array}{c}\text { Integrated CZT detector } \\
\text { (Frisch-grid) } \\
\text { Condition: E1>E2 }\end{array}$ & $\begin{array}{c}\text { Pixelated CZT } \\
\text { detector [19] }\end{array}$ \\
\hline Angular resolution $\left(^{\circ}\right)$ & $26 \pm 1.3$ & 18 \\
\hline Intrinsic efficiency & $(0.94 \pm 0.02) \times 10^{-2}$ & $1.9 \times 10^{-2}$ \\
\hline $\begin{array}{l}\text { Absolute efficiency } \\
\text { (assuming 1-m distance) }\end{array}$ & $(9.69 \pm 0.22) \times 10^{-7}$ & $3.58 \times 10^{-7}$ \\
\hline
\end{tabular}

than a single unit of our detector, and hence, the angular resolution and intrinsic efficiency of the pixelated detector is higher than those of our Frisch-grid detectors. However, the entire size of the Frisch-grid detector is larger than that of the pixelated detector, and the absolute efficiency of the Frisch-grid detector is 2.7 times larger than that of the pixelated detector.

The activity of ${ }^{133} \mathrm{Ba}(356 \mathrm{keV}),{ }^{137} \mathrm{Cs}(662 \mathrm{keV})$, and ${ }^{60} \mathrm{Co}$ $(1173,1332 \mathrm{keV})$ were, respectively, $169.5-\mathrm{kBq}, 281.6-\mathrm{kBq}$, and $7.8-\mathrm{kBq}$. The source-to-detector distance was $14.5 \mathrm{~cm}$. The background dose rate was $29.8 \mu \mathrm{R} / \mathrm{hr}$. Under these conditions, we could start to identify the point sources when the measured effective counts for ${ }^{133} \mathrm{Ba}(356 \mathrm{keV}),{ }^{137} \mathrm{Cs}(662 \mathrm{keV})$ and ${ }^{60} \mathrm{Co}$ $(1173,1332 \mathrm{keV})$ were, respectively, more than $90,30,35$, and 25 . The ratios of the maximum to the standard deviation of peripheral pixels at the 1 st iteration were 9.3, 11.1, 10.9, and 9.5 for 356-, 662-, 1173-, and 1332-keV gamma-rays, respectively.

Based on the minimum counts for image reconstruction, we estimated the required exposure time, the total area of each layer, and the number of the layers to detect radiation isotopes; the results are shown in Fig. 6. The activities of the sources were $3700-\mathrm{kBq}(100-\mu \mathrm{Ci})$, the thickness of each crystal layer was $15 \mathrm{~mm}$, and the distance between the source and detector was $10 \mathrm{~m}$. The radiation attenuation, which was proportional to the number of crystal layers, was considered in the estimation.

\section{CONCLUSIONS}

By using an array of Frisch-grid CdZnTe detectors, we developed a mini Compton camera and compared its performance with those achieved by previous researchers based on a pixelated detector. The efficiency and angular resolution of our camera were experimentally measured for various gamma-ray sources (at energies of 356-, 662-, 1137-, and 1332-keV).

Compared to the previous pixelated detector [19], the angular resolution of our camera was broader, but the absolute efficiency was high. Recently, developed pixelated detectors had a larger volume than earlier versions as the detector materials have improved [29].

From the point of view of large-scale manufacturing and maintenance, including the addition and replacement of detector units, a Frisch-grid detector has a simple, convenient, and economical structure. The Frisch-grid geometry offers flexibility in the design and layout of multi-element detector modules, plus less constraints on adapting to new design features. The price for virtual Frisch-grid devices will be reduced in comparison to other devices requiring large monolithic single 


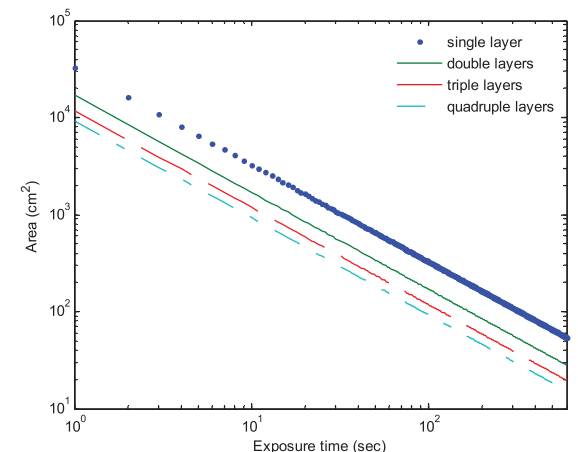

(a)

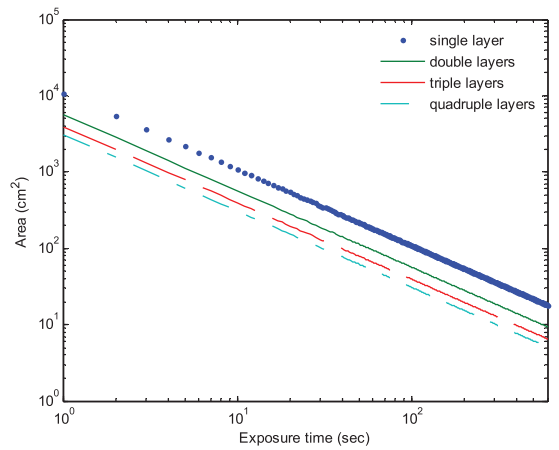

(b)

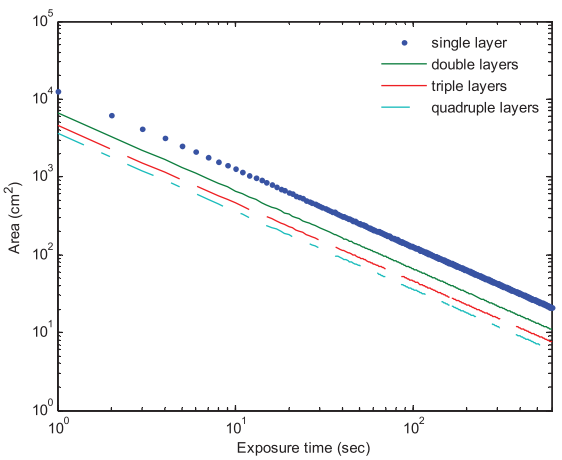

(c)

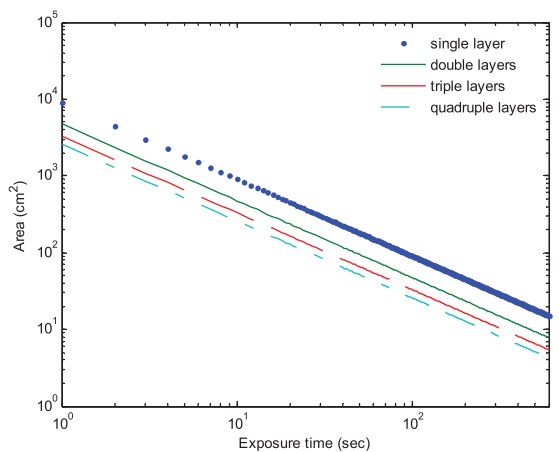

(d)

Fig. 6. Required detector area, number of layers, and exposure time to detect radiation isotopes. (a) ${ }^{133} \mathrm{Ba}(356 \mathrm{keV})$, (b) ${ }^{137} \mathrm{Cs}(662 \mathrm{keV})$, (c) ${ }^{60} \mathrm{Co}$ (1173 keV), and (d) ${ }^{60} \mathrm{Co}(1332 \mathrm{keV})$.

crystals, because the yield of acceptable material is higher than that for large, single-crystal volumes due to the relaxed constraints on material homogeneity and carrier-transport properties. It should be pointed out the prices for both virtual Frisch-grid and multi-pixel CdZnTe detectors has been steadily decreasing over the past 10 years. Furthermore, the precise cost for each design (Frisch-grid, multi-pixel, co-planar grid, hemispheric, among others) is likely to continue to decrease as the yields of suitable detector materials are further increased, thus enabling larger and thicker detectors than available today.

In further studies, we will use crystals of different thickness and apply emerging 3D positioning technology, such as the improved depth information by relying on the anode-to-cathode signal ratios or the charge drift times, and on the application of segmented sensing electrodes at the side surfaces in each single Frisch-grid detector to improve the position resolution within the detector through use of the transient signals induced on these sensing pads and Anger logic [15]. This implementation will further enhance the angular resolution of the camera, and it can be expanded to arrays of position-sensitive Frischgrid detectors.

\section{REFERENCES}

[1] T. E. Schlesinger and R. B. James, "Semiconductors and semimentals," in Semiconductors for Room Temperature Nuclear Detector Applications. San Diego, CA: Academic, 1995, vol. 43.

[2] P. N. Luke and E. E. Eissler, "Performance of CdZnTe coplanar-grid gamma-ray detectors," IEEE Trans. Nucl. Sci., vol. 43, no. 3, pp. 1481-1486, 1996.

[3] Z. He, W. Li, G. F. Knoll, D. K. Wehe, J. Berry, and C. M. Stahle, "3-D position sensitive CdZnTe gamma-ray spectrometers," Nucl. Instr. Meth. A, vol. 422, pp. 173-178, 1999.

[4] F. Zhang, Z. He, D. Xu, G. F. Knoll, D. K. Wehe, and J. E. Berry, "Improved resolution for 3-D position sensitive CdZnTe spectrometers," IEEE Trans. Nucl. Sci., vol. 51, no. 5, pp. 2427-2431, 2004.

[5] D. S. McGregor, Z. He, H. A. Seifert, D. K. Wehe, and R. A. Rojeski, "Single charge carrier type sensing with a parallel strip pseudo-frischgrid CdZnTe semiconductor radiation detector," Appl. Phys. Lett., vol. 72, pp. 792-794, 1998.

[6] W. J. McNeil, D. S. McGregor, A. E. Bolotnikov, G. W. Wright, and R. B. James, "Single-charge-carrier-type sensing with an insulated Frisch ring CdZnTe semiconductor radiation detector," Appl. Phys. Lett., vol. 84, pp. 1988-1990, 2004.

[7] A. Kargar, E. Ariesanti, and D. S. McGregor, "A comparison between spectroscopic performance of $\mathrm{HgI}_{2}$ and CdZnTe frisch collar detectors," Nucl. Technol., vol. 175, pp. 131-137, 2011.

[8] A. Kargar, M. J. Harrison, A. C. Brooks, and D. S. McGregor, "Characterization of charge collection in a CdZnTe frisch collar detector with a highly collimated ${ }^{137} \mathrm{Cs}$ Source," Nucl. Instrum. and Meth., A, vol. 620, pp. 270-278, 2010.

[9] M. J. Harrison, A. Kargar, and D. S. McGregor, "Improved techniques for the fabrication of frisch collar CdZnTe gamma ray spectrometers," IEEE Trans. Nucl. Sci., vol. 56, pp. 1671-1676, 2007.

[10] F. Zhang, C. Herman, Z. He, G. De Geronimo, E. Vernon, and J. Fried, "Characterization of the H3D ASIC readout system and $6.0 \mathrm{~cm}^{3} 3-\mathrm{D}$ position sensitive CdZnTe detectors," IEEE Trans. Nucl. Sci., vol. 59, no. 1, pp. 236-242, 2012.

[11] A. E. Bolotnikov, N. M. Abdul-Jabbar, S. Babalola, G. S. Camarda, Y. Cui, A. Hossain, E. Jackson, H. Jackson, J. R. James, A. L. Luryi, and R. B. James, "Optimization of virtual frisch-grid CdZnTe detector designs for imaging, and spectroscopy of gamma rays," in Proc. SPIE Hard X-Ray and Gamma-Ray Detector Phys. IX, Bellingham, WA, USA, 2007, vol. 6706, pp. 670603-1-670603-14.

[12] Y. Cui, A. Bolotnikov, G. Carmarda, A. Hossain, G. Yang, and R. B. James, "CZT virtual frisch-grid detector: Principles and applications," in Proc. Fifth Annu.l IEEE Long Island Systems, Applications, and Technol. Conf., Farmingdale, NY, USA, 2009.

[13] J. K. Polack, M. Hirt, J. Sturgess, N. D. Sferrazza, A. E. Bolotnikov, S. Babalola, G. S. Carmarda, Y. Cui, S. U. Egarievwe, P. M. Fochuk, R. Gul, A. Hossain, K. Kim, O. V. Kopach, L. Marchini, G. Yang, L. Xu, and R. B. James, "Variation of electric shielding on virtual frisch-grid detectors," Nucl. Instrum. Meth. Phys. Res. A, vol. 621, pp. 424-430, 2010 . 
[14] A. E. Bolotnikov, J. Butcher, G. S. Camarda, G. De Geronimo, J. Fried, R. Gul, P. M. Fochuk, M. Hamade, A. Hossain, K. H. Kim, O. V. Kopach, M. Petryk, E. Vernon, G. Yang, and R. B. James, “Array of virtual frisch-grid CZT detectors with common cathode readout for correcting charge signals and rejection of incomplete charge-collection events," IEEE Trans. Nucl. Sci., vol. 59, no. 4, pp. 1544-1551, 2012.

[15] A. E. Bolotnikov, K. Ackley, G. S. Camarda, C. Cherches, Y. Cui, G. De Geronimo, J. Fried, D. Hodges, A. Hossain, W. Lee, G. Mahler, M. Maritato, M. Petryk, U. Roy, C. Salwen, E. Vernon, G. Yang, and R. B. James, "An array of virtual frisch-grid CdZnTe detectors and a front-end application-specific integrated circuit for large-area positionsensitive gamma-ray cameras," Rev. Sci. Instrum., vol. 86, no. 073114, pp. 1-10, 2015.

[16] D. Herzo, R. Koga, W. A. Millard, S. Moon, J. Ryan, R. Wilson, A. D. Zych, and R. S. White, "A large double scatter telescope for gamma rays and neutrons," Nucl. Instrum. Methods Phys. Res. A, vol. 251, pp. 132-134, 1974

[17] M. Singh, "An electronically collimated gamma camera for single photon emission computed tomography. Part I: Theoretical considerations and design criteria," Med. Phys., vol. 10, pp. 421-427, 1983.

[18] Y. F. Du, Z. He, G. F. Knoll, D. K. Wehe, and W. Li, "Evaluation of compton scattering camera using 3-D position sensitive CdZnTe detectors," Nucl. Instrum. Methods Phys. Res. A, vol. A457, pp. 203-211, 2001.

[19] C. E. Lehner, Z. He, and F. Zhang, " $4 \pi$ compton imaging using a 3-D position-sensitive CdZnTe detector via weighted list-mode maximum likelihood," IEEE Trans. Nucl. Sci., vol. 51, no. 4, pp. 1618-1624, 2004.

[20] W. Wang, C. Wahl, J. Jaworski, and Z. He, "Maximum-likelihood deconvolution in the spatial and spatial-energy domain for events with any number of iterations," IEEE Trans. Nucl. Sci., vol. 59, no. 2, pp. 469-478, 2012.
[21] K. Vetter, M. Burks, C. Cork, M. Cunningham, D. Chivers, E. Hull, T. Krings, H. Manini, L. Michailescu, K. Nelson, D. Protic, J. Valentine, and D. Wright, "High sensitivity compton imaging with position sensitive Si and Ge detectors," Nucl. Instrum. Meth. Phys. Res. A, vol. A579, pp. 363-366, 2007.

[22] G. J. Schmid, D. A. Beckedahl, J. E. Kammeraad, J. J. Blair, K. Vetter, and A. Kuhn, "Gamma-ray compton camera imaging witha segmented HPGe," Nucl. Instrum. Meth. Phys. Res. A, vol. A459, pp. 565-576, 2001.

[23] H. H. Barrett, T. White, and L. C. Parra, "List-mode likelihood," $J$. Opt. Soc. Amer., vol. A14, pp. 2914-2923, 1997.

[24] L. Parra and H. H. Barrett, "List-mode likelihood: EM algorithm and image quality estimation demonstrated on 2-D PET," IEEE Trans. Med. Imag., vol. 17, no. 2, pp. 228-235, 1998.

[25] S. J. Wilderman, N. H. Clinthorne, J. A. Fessler, and W. Les Rogers, "List-mode maximum likelihood reconstruction of compton scatter camera image in nuclear medicine," in Proc. IEEE Nucl. Sci. Symp., Toronto, Ontario, Canada, 1999, vol. 3, pp. 1716-1720.

[26] W. Lee, A. Jo, T. Lee, and D. Chu, "Monte carlo simulation on the $4 \pi$ field of a view compact compton camera by using scintillators," $J$. Korean Phys. Soc., vol. 56, no. 1, pp. 20-27, 2010.

[27] G. F. Knoll, Radiation Detection and Measurement, 3rd ed. New York, NY: Wiley, 2000, p. 483.

[28] Y. F. Du, "Development of a prototype compton scattering camera using 3-d position sensitive CZT detectors," Ph.D. dissertation, Dept. Nucl. Eng. and Radiological Sci., Univ. of Michigan, Ann Arbor, 2001.

[29] Y. Z. Boucher, J. Jaworski, W. Kaye, F. Zhang, and Z. He, "Results from testing of $1453 \mathrm{D}$ position-sensitive, pixelated CdZnTe detectors," IEEE Trans. Nucl. Sci., vol. 59, no. 6, pp. 3332-3338, 2012. 